\title{
Cost oriented competing processes - a new handling of assignment problems
}

\author{
Jens Starke \\ University of Stuttgart, II. Institute of Theoretical Physics \\ Pfaffenwaldring 57/III, D-70550 Stuttgart, Germany. \\ Tel: +49711 685-4932. Fax: +49711685-4902. \\ e-mail: jens@theo2.physik.uni-stuttgart.de
}

\begin{abstract}
The method of Cost Oriented Competing Processes (COCP) is a new approach to handle assignment problems and combinatorial optimization problems. The COCP method works with a system of nonlinear coupled ordinary differential equations with suitable stable points. In contrast to many other techniques, this method can easily be generalized and adapted to different assignment problems, even to those of higher order and with additional constraints. In assignment problems of higher order, the single costs which have to be considered for the total costs depend on several decisions instead of only one decision as in linear assignment problems. The handling of those higher order assignment problems is important for several scheduling problems such as manufacturing planning and time table planning.
\end{abstract}

Keywords

Combinatorial optimization, assignment problems, quadratic assignment problems (QAP), assignment problems of higher order, scheduling, selforganization, manufacturing planning, neural networks

\section{LINEAR ASSIGNMENT PROBLEMS}

In an assignment problem (see e.g. (Eiselt et al., 1987), (Zimmermann, 1990)) there are decision variables $x_{i j} \in\{0,1\}$. The aim of linear assignment problems is to minimize

$\sum_{i, j} c_{i j} \cdot x_{i j}$ 
with the $\cos t s c_{i j}$ and with respect to the constraints

$$
\begin{aligned}
& \sum_{i} x_{i j}=1 \quad \forall j \in\{1, \ldots, N\} \quad \text { and } \\
& \sum_{j} x_{i j}=1 \quad \forall i \in\{1, \ldots, N\} .
\end{aligned}
$$

The matrix $\left(x_{i j}\right)$ is called the permutation matrix. The row index $i$ indicates e.g. the number of a particular worker and the column index $j$ relates to a particular job. The costs $c_{i j} \in \mathbb{R}$ have to be incurred if the $i$-th worker is assigned to the $j$-th job, i.e. if and only if $x_{i j}=1$.

\subsection{Handling of assignment problems using COCP}

The Cost Oriented Competing Processes handle assignment problems and combinatorial optimization problems by using a system of nonlinear coupled ordinary differential equations with suitable stable points. In other words: the discrete problem is handled with a method for continuous variables. This is possible because the positions of the stable points of the differential equations of the COCP are discrete.

First, a linear transformation of the cost array is made to change the minimizing problem to a maximizing problem:

$$
\xi_{i j}\left(t_{0}\right)=a-b \cdot c_{i j} \quad \text { with } \quad a, b>0 \quad \text { and } \quad \xi_{i j}\left(t_{0}\right) \geq 0 \quad \forall i, j .
$$

The condition $\xi_{i j}\left(t_{0}\right) \geq 0 \quad \forall i, j$ ensures a nonnegative output of the method. Because of numerical reasons $a$ and $b$ are chosen in a way that $\xi_{i j} \leq 1 \quad \forall i, j$. If this condition is not fulfilled, the values of the following differential equations (7) will become too large for a numerical treatment. Second, the variables $\xi_{i j}$ are submitted to a dynamic competing process. The stable points $\xi_{i j}^{*}$ of the dynamics of the COCP fulfill the constraints $\xi_{i j}^{*} \in$ $\{0,1\}$ as well as the equations (2) and (3) for the unknown decision variables $x_{i j}$. Thus for any stable points $\xi_{i j}^{*}$ the condition of a permutation matrix is fulfilled. Therefore, in the last step, the stable point in which the dynamic process ends can be assigned to the decision variables $x_{i j} \in\{0,1\}$. The nonvanishing elements $\xi_{i j}^{*}$ with $\xi_{i j}^{*}=1$ correspond to the final choice of positive decisions. The nonexistence of further stable points can be proved. Under consideration of the constraints, the dynamic competing processes prefer larger $\xi_{i j}$ (i.e. smaller costs $c_{i j}$ ) to change them to ' 1 ' and the others to ' 0 '. This guarantees that the decisions $x_{i j}$ are made by using a selforganizing process, selecting the lowest costs with respect to the constraints. The procedure of the COCP can be illustrated as

$c_{i j} \longrightarrow \xi_{i j}\left(t_{0}\right) \longrightarrow \xi_{i j}(t) \longrightarrow \xi_{i j}^{*} \longrightarrow x_{i j}$

These competing processes are realized with a system of nonlinear coupled differential equations. Similar to the equation of motion of the synergetic computer (Haken, 1990), the dynamical system can be derived in a vivid manner by using a potential function

$$
V=-\frac{1}{2} \sum_{k} \sum_{l} \xi_{k l}^{2}+\frac{1}{4} \sum_{k} \sum_{l} \xi_{k l}^{4}
$$




$$
+\frac{1}{2} \sum_{k} \sum_{l} \sum_{\substack{k^{\prime} \\ k^{\prime} \neq k}} \xi_{k l}^{2} \xi_{k^{\prime} l}^{2}+\frac{1}{2} \sum_{k} \sum_{l} \sum_{\substack{l^{\prime} \\ l^{\prime} \neq l}} \xi_{k l}^{2} \xi_{k l^{\prime}}^{2} \quad \text { with } \quad k, k^{\prime}, l, l^{\prime}=1, \ldots, N
$$

and a gradient descent method

$$
\dot{\xi}_{i j}=-\frac{\partial V}{\partial \xi_{i j}} \text {. }
$$

The potential function $V$ has minima, i. e. stable points, if and only if the constraints of a permutation matrix are fulfilled. A proof by examination of the Hesse matrix $\frac{\partial^{2} V}{\partial \xi_{i j} \partial \xi_{k l}}$ can be found in (Starke, 1994). The negative quadratic terms of the potential function (5) and the following terms of order 4 cause a stable point for $\xi_{i j}=1 \quad \forall i, j$ only. The last two terms prevent the case of more than one nonvanishing element of the matrix $\left(\xi_{i j}\right)$ in one row or column by putting 'hills' in these areas. Hereby the former stable point is replaced by stable points next to the 'hill'. Using (6) and the potential function (5) the equation of motion can be derived to

$$
\dot{\xi}_{i j}=\xi_{i j}+3 \xi_{i j}^{3}-2 \xi_{i j} \sum_{k} \xi_{k j}^{2}-2 \xi_{i j} \sum_{l} \xi_{i l}^{2}
$$

The potential function (5) has some saddlepoints. To avoid the persistence at a saddelpoint one has to add some noise to the gradient descent method (6).

A simulation example of a linear assignment problem using the COCP is shown in figure 1. The figure shows 4 time steps of the dynamic evolution of the matrix $\left(\xi_{i j}\right)$. The size

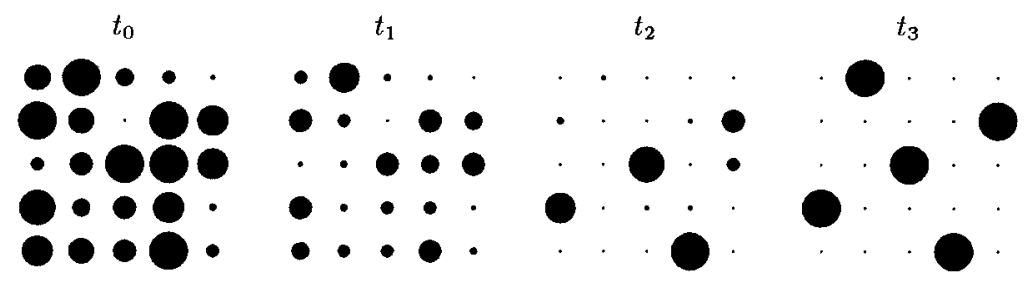

Figure 14 time steps of a simulation of the COCP handling a linear assignment problem.

of the circles in figure 1 is proportional to the size of the dynamic variables $\xi_{i j}$. In the following the cost matrix $\left(c_{i j}\right)$ and the rounded initial values $\left(\xi_{i j}\left(t_{0}\right)\right)$ of the simulation example are shown:

$$
\left(c_{i j}\right):=\left(\begin{array}{rrrrr}
8 & 3 & 11 & 13 & 16 \\
2 & 8 & 17 & 2 & 7 \\
12 & 9 & 4 & 4 & 6 \\
5 & 11 & 9 & 7 & 14 \\
6 & 8 & 9 & 3 & 13
\end{array}\right) \quad \text { and } \quad\left(\xi_{i j}\left(t_{0}\right)\right)=\left(\begin{array}{rrrrr}
0.6 & 0.9 & 0.4 & 0.3 & 0.1 \\
1.0 & 0.6 & 0.0 & 1.0 & 0.7 \\
0.3 & 0.5 & 0.9 & 0.9 & 0.7 \\
0.8 & 0.4 & 0.5 & 0.7 & 0.2 \\
0.7 & 0.6 & 0.5 & 0.9 & 0.3
\end{array}\right)
$$


The result of the simulation of the COCP is identical with the result in (Zimmermann, 1990) who applied the hungarian method.

\section{Additional constraints}

Additional constraints can easily be considered by adding further potential terms to $V$ which remove the unwanted stable points of the differential equations. The case of two nonvanishing elements $\xi_{i j}$ and $\xi_{k l}$ can be prevented by an additional potential term

$V^{\prime}=\alpha \cdot \xi_{i j}^{2} \cdot \xi_{k l}^{2}$

The constant $\alpha>0$ has to be chosen in a suitable way so that the 'hill' fills up the unwanted minima sufficiently. This has to be checked by a stability analysis. By using the additional potential term

$V^{\prime \prime}=\beta \cdot \xi_{k_{1} k_{2}}^{2} \cdot \xi_{k_{3} k_{4}}^{2} \cdot \ldots \cdot \xi_{k_{2 m-1} k_{2 m}}^{2}$

with suitable $\beta>0$, it is even possible to prevent the specific combination of the decisions $x_{k_{1} k_{2}}=1, \ldots, x_{k_{2 m-1} k_{2 m}}=1$.

The resulting potential function which has to be used for the equation of motion (6) is

$\tilde{V}=V+V^{\prime}$ or respectively $\quad \tilde{V}=V+V^{\prime \prime}$.

An example of preventing specific combinations of decisions is given in (Starke, 1994) for the TSP.

\section{Selection processes of biological macromolecules}

The evolutionary processes of biological macromolecules (Eigen, 1971), (Eigen and Schuster, 1977), (Eigen and Schuster, 1978) are special cases of these competing processes. In contrast to the differential equations in the papers cited above, each variable $\xi_{i j}$ of the Cost Oriented Competing Processes competes with different partners.

\subsection{Competing process between two elements}

The preference of the largest elements $\xi_{i j}$ can easily be shown in the following example. The competing process between the two elements $\xi_{i j}$ and $\xi_{i r}$ in row $i$ of matrix $\xi$ is given by the following two differential equations:

$$
\begin{aligned}
& \dot{\xi}_{i j}=\xi_{i j} \cdot\left(1+3 \xi_{i j}^{2}-2 \sum_{k} \xi_{k j}^{2}-2 \sum_{l} \xi_{i l}^{2}\right) \\
& \dot{\xi}_{i r}=\xi_{i r} \cdot\left(1+3 \xi_{i r}^{2}-2 \sum_{k} \xi_{k r}^{2}-2 \sum_{l} \xi_{i l}^{2}\right)
\end{aligned}
$$

In the special case of $\sum_{k} \xi_{k j}^{2}=\sum_{k} \xi_{k r}^{2}$ from $\xi_{i j}>\xi_{i r}$, one gets the result $\dot{\xi}_{i j}>\dot{\xi}_{i r}$ because of the strictly monotonouse right hand side of the differential equations (10) and (11). 
That means that the larger variable $\xi_{i j}$ grows faster $\left(\dot{\xi}_{i j}>0\right)$ or decays slower $\left(\dot{\xi}_{i j}<0\right)$. Because of this fact and because of the special position of the stable points, the largest element $\xi_{i j}$ of row $i$ will grow to ' 1 ' and all other decay to ' 0 '.

\subsection{Possibilities of intervention for the human decision-maker}

The COCP provides two possibilities of intervention for the decision-maker. First, general rules or experiences of the human decision-maker can be considered by using further potential terms in the form of (8) or (9) to prevent specific decisions. With this it is even possible to prevent a specific combination of several decisions. Second, single changes of the problem configuration, like missing material or broken machines, can be considered by initializing the corresponding elements of the matrix $\left(\xi_{i j}\right)$ with ' 0 ' to prevent or ' 1 ' to enforce a particular assignment.

\subsection{Neural network interpretation}

Similar to (Haken, 1990), (Baird, 1990) and (Hirsch and Baird, 1995) the differential equations of the Cost Oriented Competing Processes can be treated as a neural network of higher order. The equation of motion of the COCP can be written in the form

$\dot{\xi}_{i j}=\sum_{k, l} T_{i j k l} \cdot \xi_{k l}+\sum_{k, l, m, n} J_{i j k l m n} \cdot \xi_{i j} \xi_{k l} \xi_{m n}$

The constants $J_{i j k i m n}$ of the higher order terms in the equation of motion can be interpreted as higher order couplings. In the COCP these higher order couplings and the couplings $T_{i j k l}$ are chosen in a way so that the final output $\xi_{i j}^{*}$, i. e. the stable points, correspond to decision variables $x_{i j} \in\{0,1\}$ with respect to the constraints of the assignment problem.

\section{ASSIGNMENT PROBLEMS OF HIGHER ORDER}

The principles of the COCP explained in the section on linear assignment problems can be transferred to assignment problems of higher order by using an appropriate number of dimensions for the dynamic variables.

\subsection{Quadratic assignment problems (QAP)}

Handling quadratic assignment problems (QAP), one has to minimize

$\sum_{i, j, k, l} c_{i j k l} \cdot x_{i j} \cdot x_{k l}$

with respect to the constraints $x_{i j} \in\{0,1\}$,

$\sum_{i} x_{i j}=1 \quad \forall j \in\{1, \ldots, N\} \quad$ and $\quad \sum_{j} x_{i j}=1 \quad \forall i \in\{1, \ldots, N\}$. 
The costs $c_{i j k l}$ have to be incurred for the total costs if and only if $x_{i j}=1$ and $x_{k l}=1$, i. e. if and only if both decisions are chosen positive. In this case, the Cost Oriented Competing Processes are applied to the 4-dimensional array $\left(c_{i j k l}\right)$. For this reason the dynamic variable $\xi_{i j k l}$ is 4-dimensional, too. In order to calculate the initial values $\xi_{i j k i}\left(t_{0}\right)$ the linear transformation (4) is used again. Then the equation of motion is

$$
\begin{aligned}
\dot{\xi}_{i j k l} & =-\frac{\partial V}{\partial \xi_{i j k l}} \\
& =\xi_{i j k l}+7 \xi_{i j k l}^{3}-2 \xi_{i j k l}\left(\sum_{i^{\prime}, j^{\prime}, k^{\prime}} \xi_{i^{\prime} j^{\prime} k^{\prime} l}^{2}+\sum_{i^{\prime}, j^{\prime}, l^{\prime}} \xi_{i^{\prime} j^{\prime} k l^{\prime}}^{2}+\sum_{i^{\prime}, k^{\prime}, l^{\prime}} \xi_{i^{\prime} j k^{\prime} l^{\prime}}^{2}+\sum_{j^{\prime}, k^{\prime}, l^{\prime}} \xi_{i j^{\prime} k^{\prime} l^{\prime}}^{2}\right) .
\end{aligned}
$$

The positions of the stable points of this dynamics ensure that $\xi_{i j k l} \in\{0,1\}$ and

$$
\sum_{i, j, k} \xi_{i j k l}^{*}=1 \quad \forall l, \ldots, \sum_{j, k, l} \xi_{i j k l}^{*}=1 \quad \forall i .
$$

The decision variables $x_{i j}$ are obtained by projecting the array $\xi_{i j k l}^{*}$ onto those two dimensions belonging to the decision variables $x_{i j}$ with

$x_{i j .}=\sum_{k, l} \xi_{i j k l}^{*}$

In time dependent optimization problems, the other dimensions can belong to previous decisions. The constraints of the assignment problem are fulfilled because of

$$
\begin{aligned}
\sum_{i} x_{i j} & =\sum_{i} \sum_{k, l} \xi_{i j k l}^{*}=1 \quad \forall j \\
\sum_{j} x_{i j} & =\sum_{j} \sum_{k, l} \xi_{i j k l}^{*}=1 \quad \forall i .
\end{aligned}
$$

\subsection{Assignment problems of order $n$}

In assignment problems of order $n$ one has to minimize

$\sum_{k_{1}, k_{2}, \ldots, k_{2 n}} c_{k_{1}, k_{2}, \ldots, k_{2 n}} \cdot x_{k_{1} k_{2}} \cdot x_{k_{3} k_{4}} \cdot \ldots \cdot x_{k_{2 n-1} k_{2 n}}$

with respect to the constraints $x_{k_{1} k_{2}} \in\{0,1\}$,

$\sum_{k_{2}} x_{k_{1} k_{2}}=1 \quad \forall k_{2} \in\{1, \ldots, N\} \quad$ and $\quad \sum_{k_{2}} x_{k_{1} k_{2}}=1 \quad \forall k_{1} \in\{1, \ldots, N\}$

The costs $c_{k_{1}, \ldots, k_{2 n}}$ have to be considered for the total costs if and only if all of the decision variables $x_{k_{1} k_{2}}, x_{k_{3} k_{1}}, \ldots, x_{k_{2 n-1} k_{2 n}}$ are equal 1 , i. e. if and only if all decisions are chosen positive. In this case, the Cost Oriented Competing Processes are applied to 
the $2 \mathrm{n}$-dimensional array $\left(c_{k_{1}, \ldots, k_{2 n}}\right)$. As in the quadratic assignment problem, the linear transformation (4) is used here again. The result of the equation of motion is

$$
\begin{aligned}
\dot{\xi}_{k_{1}, \ldots, k_{2 n}}= & -\frac{\partial V}{\partial \xi_{k_{1}, \ldots, k_{2 n}}} \\
= & \xi_{k_{1}, \ldots, k_{2 n}}+(4 n-1) \cdot \xi_{k_{1}, \ldots, k_{2 n}}^{3} \\
& -2 \xi_{k_{1}, \ldots, k_{2 n}}\left(\sum_{k_{1}^{\prime}, \ldots, k_{2 n-1}^{\prime}} \xi_{k_{1}^{\prime}, \ldots, k_{2 n-1}^{\prime}, k_{2 n}}^{2}+\ldots+\sum_{k_{2}^{\prime}, \ldots, k_{2 n}^{\prime}} \xi_{k_{1}, k_{2}^{\prime}, \ldots, k_{2 n}^{\prime}}^{2}\right) .
\end{aligned}
$$

The decision variables $x_{k_{1} k_{2}}$ can be derived as in the case of the quadratic assignment problem by projecting the $n$ dimensional array $\xi_{k_{1}, k_{2}, \ldots, k_{2 n}}^{*}$ onto the two dimensions which belong to the decision variables $x_{k_{1} k_{2}}$.

The considerations of competing processes between two elements, the possibilities of intervention for the decision maker and the neural network interpretation in the section of linear assignment problems can be directly transferred.

\section{EXAMPLE OF INDUSTRIAL ASSIGNMENT PROBLEMS OF HIGHER ORDER}

Assignment problems of quadratic or higher order often occur in manufacturing planning. The job scheduling of a weaving mill is a good example. In this problem, many $(10-200)$ similar machines and about 4 times as many jobs with many possible assignments exist. The costs to be considered are setup costs, tardiness costs (for late jobs) and prohibition costs because of limited machine capacities and missing material. If one considers only one preceding job for the calculation of the costs, the method of the Cost Oriented Competing Processes can be adapted to this problem by calculating a 3-dimensional cost array to initialize the competing processes and using a linkage condition between the time steps (Starke and Berkemer, 1995). Additional dimensions allow the consideration of further preceding jobs in the optimization of the scheduling.

\section{CONCLUSIONS AND OUTLOOK}

For industrial applications it is remarkable that the algorithm of the Cost Oriented Competing Processes can easily be adapted to higher order assignment problems with specific constraints. It is possible to prohibit or enforce particular decisions. Therefore the experience of human decision-makers can be used additionally and one can pay attention to sudden changes of the constraints of the problem configuration.

Moreover, the COCP can be computed in a highly parallel way. Because of the close relationship of the equations of motion proposed here and of these of some physical systems like e.g. the laser (Haken, 1983), a hardware realization of the COCP should be possible. First theoretical steps with hardware realizations of some simpler selection processes are made with semiconductors and lasers in (Schindel, 1993) and (Beckert, 1994). 


\section{REFERENCES}

Baird, B. (1990) A Learning Rule for CAM Storage of Continuous Periodic Sequences, in Proceedings of the International Conference on Neural Networks, San Diego, June 1990 , III.

Beckert, S. (1994) Modenselektion beim Laser zur Realisierung des Synergetischen Computers. Dissertation, University of Stuttgart.

Eigen, M. (1971) Selforganization of Matter and the Evolution of Biological Macromolecules. Die Naturwissenschaften, 58, 465-523.

Eigen, M. and Schuster, P. (1977) The Hypercycle - Part A: Emergence of the Hypercycle. Die Naturwissenschaften, 64, 541-65.

Eigen, M. and Schuster, P. (1978) The Hypercycle - Part B: The Abstract Hypercycle. Die Naturwissenschaften, 65, 7-41.

Eiselt, H. A., Pederzoli, G. and Sandblom, C.-L. (1987) Operations Research: Continuous Optimization Models. Walter de Gruyter, Berlin New York.

Haken, H. (1983) Synergetics, An Introduction. Springer-Verlag, Berlin Heidelberg.

Haken, H. (1990) Synergetic Computers and Cognition - A Top-Down Approach to Neural Nets. Springer Verlag, Berlin Heidelberg.

Hirsch, M. W. and Baird, B. (1995) Computing with dynamic attractors in neural networks. BioSystems, 34, 173-95.

Schindel, M. (1993) Theorie eines Halbleitersystems zur Realisierung der Ordnungsparameterdynamik eines Synergetischen Computers. Dissertation, University of Stuttgart.

Starke, J. (1994) Vergleichende Untersuchung lernfähiger Systeme. Diploma thesis, University of Stuttgart.

Starke, J. and Berkemer, R. (1995) Manufacturing Planning with Cost Oriented Competing Processes - Example of a Weaving Mill, will be published.

Zimmermann, W. (1990) Operations Research. Oldenbourg Verlag, München Wien. 